PROCEEDINGS OF THE

AMERICAN MATHEMATICAL SOCIETY

Volume 131, Number 1, Pages 165-174

S 0002-9939(02)06826-0

Article electronically published on June 12, 2002

\title{
A FOURIER SERIES FORMULA FOR ENERGY OF MEASURES WITH APPLICATIONS TO RIESZ PRODUCTS
}

\author{
KATHRYN E. HARE AND MARIA ROGINSKAYA \\ (Communicated by David Preiss)
}

\begin{abstract}
In this paper we derive a formula relating the energy and the Fourier transform of a finite measure on the $d$-dimensional torus which is similar to the well-known formula for measures on $\mathbb{R}^{d}$.

We apply the formula to obtain estimates on the Hausdorff dimension of Riesz product measures. These give improvements on the earlier, classical results which were based on completely different techniques.
\end{abstract}

\section{INTRODUCTION}

Potential theoretic techniques have been quite useful in geometric measure theory for studying the behaviour of measures and sets in $\mathbb{R}^{d}$. There is a well-known and important relationship between the $t$-energy of a measure, $I_{t}(\mu)$, and its Fourier transform

$$
I_{t}(\mu) \equiv \iint \frac{d \mu(x) d \mu(y)}{|x-y|^{t}}=c_{t, d} \int|x|^{t-d}|\widehat{\mu}(x)|^{2} d x
$$

and this has been used to study a variety of things, including the Hausdorff dimension of projections and intersections of sets, distance sets, and the average rate of decay of the Fourier transform (cf. [1], [9], [10], [13] and the references cited therein).

Here we derive a similar formula relating the energy and discrete Fourier transform of a measure on the $d$-dimensional torus

$$
I_{t}(\mu) \sim|\widehat{\mu}(0)|^{2}+\sum_{z \in \mathbb{Z}^{d} \backslash\{0\}}|z|^{t-d}|\widehat{\mu}(z)|^{2},
$$

and apply the formula to study the dimensions of Riesz product measures.

Riesz product measures are typically singular to Lebesgue measure so it is of interest to determine their Hausdorff dimension. This problem was initially investigated in a remarkable paper by Peyrière [11]. One consequence of our energy formula is that if $\left\{n_{k}\right\}$ is a lacunary sequence of integers satisfying $n_{k+1} / n_{k} \geq 3$,

Received by the editors August 17, 2001.

2000 Mathematics Subject Classification. Primary 28A12, 42A55.

Key words and phrases. energy, Hausdorff dimension, Riesz products.

This research was done while the first author enjoyed the hospitality of the Department of Mathematics at Göteborg University and Chalmers Institute of Technology. It was supported in part by NSERC and the Swedish Natural Sciences Research Council.

(C)2002 American Mathematical Society 
then the Riesz product measure on the torus, $\mu=\prod_{j=1}^{\infty}\left(1+\operatorname{Re} a_{j} e^{i n_{j} x}\right)$, has Hausdorff dimension at least

$$
1-\limsup _{k \rightarrow \infty}\left(\sum_{j=1}^{k-1}\left|a_{j}\right|^{2} /\left(2 \log n_{k}\right)\right) .
$$

This improves upon (some of) Peyrière's work [1] and a subsequent paper of Brown, Moran and Pearce [2] where it was shown, using other techniques, that the Hausdorff dimension of a more restricted class of Riesz products was at least $1-\lim \sup \left(\sum_{j=1}^{k-1}\left|a_{j}\right| / \log n_{k}\right)$.

Similar results can be obtained for the dimensions of multivariable Riesz products in $\mathbb{T}^{d}$ and $\mathbb{R}^{d}$. These again are sharper and apply to a larger class of measures than the earlier results of Peyrière.

\section{Energy and the Fourier transform}

By a measure we mean a finite, positive Borel measure on a metric space. The $t$-energy of the measure $\mu, I_{t}(\mu)$, is defined as

$$
I_{t}(\mu) \equiv \iint \frac{d \mu(x) d \mu(y)}{\operatorname{dist}(x, y)^{t}} .
$$

Our interest is in the metric spaces $\mathbb{R}^{d}$ with the usual Euclidean metric $\operatorname{dist}(x, y)$ $=|x-y|$, and the $d$-dimensional torus, $\mathbb{T}^{d}$, which we view as either $[-1 / 2,1 / 2]^{d}$ or $[-\pi, \pi]^{d}$ with the usual identification (depending on which is more convenient). The torus has a group structure and when we write $x-y$ for $x, y \in \mathbb{T}^{d}$ it should be understood that the binary operation is the group operation. The metric we consider on $\mathbb{T}^{d}$ is the usual notion of distance on the torus and we denote this metric by $d_{\mathbb{T}}(x, y)$ to distinguish it from the metric on $\mathbb{R}^{d}$.

The Hausdorff dimension of a measure $\mu$ is defined as

$$
\operatorname{dim}_{H} \mu \equiv \inf \left\{\operatorname{dim}_{H} E: E \text { is a Borel set with } \mu(E)>0\right\} .
$$

For properties of the Hausdorff dimension of a measure see [4, ch. 10].

We will say that $t$ is the energy dimension of $\mu$ if

$$
t=\sup \left\{s: I_{s}(\mu)<\infty\right\} .
$$

If $I_{t}(\mu)<\infty$, then $\operatorname{dim}_{H} \mu>t$ (cf. [3, 4.3]); thus, the Hausdorff dimension of a measure is always at least the energy dimension.

Although the Hausdorff and energy dimensions are not always equal, for many measures they are. For example, if $\mu$ is the Hausdorff measure on the classical middle-third Cantor set, then $\operatorname{dim}_{H} \mu=\log 2 / \log 3 \equiv s_{0}$. Since $\mu(B(x, r)) \leq c r^{s_{0}}$ for all $x$, it can be shown that $I_{t}(\mu)<\infty$ for all $t<s_{0}$, and hence the two dimensions coincide.

There is a very useful relationship between the energy and Fourier transform of a measure on $\mathbb{R}^{d}$ which can be derived from Parseval's theorem and the well-known fact that if $f(x)=|x|^{-t}$ for $x \in \mathbb{R}^{d}$, then $\widehat{f}(z)=c_{t, d}|z|^{t-d}$ for some constant $c_{t, d}$, namely,

$$
I_{t}(\mu)=c_{t, d} \int|x|^{t-d}|\widehat{\mu}(x)|^{2} d x .
$$

A good explanation of the derivation of this formula and some examples of applications are given in [조, ch. 12]. 
It is natural to ask if there is a similar formula relating the energy and the (discrete) Fourier transform of a measure on the $d$-dimensional torus. When $d=1$, one can obtain a relationship by using Parseval's theorem and the fact (see [14] p. 70]) that

$$
\sum_{n=1}^{\infty} n^{s-1} \cos n x=c_{s}|x|^{-s}+O(1) \text { for } 0<s<1
$$

By finding a suitable kernel on $\mathbb{T}^{d}$ we will obtain a formula for $d>1$, as well.

Note that, in what follows, when we write $f \sim g$ we will mean there are positive constants $a, b$ such that $a g \leq f \leq b g$.

Lemma 2.1. Let $0<t<d$. There is a function $F_{t}$ defined on $\mathbb{T}^{d}$ and $a C^{\infty}$ function $\phi$ defined on $\mathbb{R}^{d}$ which have the following properties:

(1) $F_{t}$ is positive, integrable, continuous except at the origin and has Fourier coefficients satisfying $\widehat{F}_{t}(z) \sim|z|^{t-d}$ for $z \in \mathbb{Z}^{d}, z \neq 0$;

(2) $\phi$ is positive, bounded on $\mathbb{R}^{d}$ and bounded away from zero on $\mathbb{T}^{d}$; and

(3) $F_{t}(x)-\phi(x)|x|^{-t}$ is positive and bounded on $\mathbb{T}^{d}$ independently of $t$.

Proof. We will take $\mathbb{T}^{d}=[-1 / 2,1 / 2]^{d}$. Let $\psi_{1}$ be any nonnegative, $C^{\infty}\left(\mathbb{R}^{d}\right)$ test function supported on $B(0,1 / 4)$ (the ball centered at the origin, with radius $1 / 4$ ) satisfying $\psi_{1}(0)>0$. Set $\psi(x)=\psi_{1}(x)+\psi_{1}(-x)$ and let $\phi_{1}$ be the inverse Fourier transform of $\psi$. Then $\phi_{1}^{2}$ is nonnegative and strictly positive on a neighbourhood of 0 . The function $\phi_{1}^{2} * \phi_{1}^{2}$ is a positive, $C^{\infty}$ function which decays rapidly and whose Fourier transform, $(\psi * \psi)^{2}$, is a nonnegative function which is supported on $B(0,1 / 2)$ and is positive at zero. For $\phi$ take a suitable dilate of $\phi_{1}^{2} * \phi_{1}^{2}$ which is bounded away from zero on the torus. The boundedness and rapid decay of $\phi$ ensure that $f_{t}(x) \equiv \phi(x)|x|^{-t}$ is a summable function on $\mathbb{R}^{d}$ and thus we can form the periodic function

$$
F_{t}(x) \equiv \sum_{a \in \mathbb{Z}^{d}} f_{t}(x+a)=\sum_{a \in \mathbb{Z}^{d}} \phi(x+a)|x+a|^{-t}
$$

We consider $F_{t}$ as a function on the torus; it is clearly positive, integrable and its Fourier coefficients coincide with the Fourier transform of $f_{t}$ on the integer lattice. As $\widehat{f_{t}}=\widehat{\phi} * \widehat{|\cdot|^{-t}}$ we have

$$
\widehat{F}_{t}(z)=\widehat{f}_{t}(z)=c_{t, d} \int_{\mathbb{R}^{d}} \frac{\widehat{\phi}(y)}{|z-y|^{d-t}} d y=c_{t, d} \int_{B(0,1 / 2)} \frac{\widehat{\phi}(y)}{|z-y|^{d-t}} d y \text { for } z \in \mathbb{Z}^{d}
$$

with the latter equality arising because $\widehat{\phi}$ is supported on $B(0,1 / 2)$. If, in addition to being in the integer lattice, $z \neq 0$, then $|z-y| \sim|z|$ when $y \in B(0,1 / 2)$. As $\widehat{\phi}$ is bounded away from zero in a neighbourhood of the origin, it follows that $\widehat{F}_{t}(z) \sim|z|^{t-d}$.

The function $F_{t}(x)-\phi(x)|x|^{-t}$ is bounded independently of $t$ because of the rapid decay of $\phi ; F_{t}$ is continuous except at 0 for similar reasons. 
Theorem 2.2. Let $0<t<d$. There are constants $a, b>0$, depending on $t, d$ such that if $\mu$ is any finite, positive Borel measure on $\mathbb{T}^{d}$, then

$$
\begin{aligned}
& a\left(|\widehat{\mu}(0)|^{2}+\sum_{z \in \mathbb{Z}^{d} \backslash\{0\}}|z|^{t-d}|\widehat{\mu}(z)|^{2}\right) \\
& \leq I_{t}(\mu) \leq b\left(|\widehat{\mu}(0)|^{2}+\sum_{z \in \mathbb{Z}^{d} \backslash\{0\}}|z|^{t-d}|\widehat{\mu}(z)|^{2}\right) .
\end{aligned}
$$

Proof. Choose the functions $\phi$ and $F_{t}(x)$ found in the lemma and set $g_{t}(x)=F_{t}(x)-$ $\phi(x)|x|^{-t}$. Since $\phi$ is bounded away from zero on the torus, $I_{t}(\mu)$ is comparable to

$$
\int_{\mathbb{T}^{d}} \int_{\mathbb{T}^{d}} \frac{\phi(x-y)}{|x-y|^{t}} d \mu(x) d \mu(y)=\int_{\mathbb{T}^{d}} \int_{\mathbb{T}^{d}}\left(F_{t}-g_{t}\right)(x-y) d \mu(x) d \mu(y) .
$$

Let $\psi$ be a nonnegative, continuous function defined on $\mathbb{T}^{d}$, supported on $[-1 / 6,1 / 6]^{d}$ and satisfying $\int \psi=1$ and $\widehat{\psi} \geq 0$. Suppose $\left\{\psi_{\varepsilon}\right\}_{\varepsilon>0}$ is an approximate identity where $\psi_{\varepsilon}$ is the function supported on $[-\varepsilon / 6, \varepsilon / 6]^{d}$ given by $x \mapsto \varepsilon^{-d} \psi(x / \varepsilon)$.

As $\psi_{\varepsilon} * F_{t}$ is a continuous function on the torus, Parseval's theorem gives

$$
\iint\left(\psi_{\varepsilon} * F_{t}\right)(x-y) d \mu(x) d \mu(y)=\sum_{z \in \mathbb{Z}^{d}} \widehat{\psi_{\varepsilon}}(z) \widehat{F}_{t}(z)|\widehat{\mu}(z)|^{2}
$$

and this converges to $\sum_{z \in \mathbb{Z}^{d}} \widehat{F}_{t}(z)|\widehat{\mu}(z)|^{2}$ as $\varepsilon \rightarrow 0$ since $\widehat{\psi_{\varepsilon}}(z) \rightarrow 1$ pointwise from below. By Fatou's lemma

$$
\iint F_{t}(x-y) d \mu(x) d \mu(y) \leq \liminf _{\varepsilon \rightarrow 0} \iint \psi_{\varepsilon} * F_{t}(x-y) d \mu(x) d \mu(y),
$$

and thus $I_{t}(\mu)$ is bounded above by some multiple of

$$
\sum_{z \in \mathbb{Z}^{d} \backslash\{0\}}|z|^{t-d}|\widehat{\mu}(z)|^{2}+\widehat{F}_{t}(0)|\widehat{\mu}(0)|^{2},
$$

verifying the right-hand inequality.

The left-hand inequality is trivially true if $I_{t}(\mu)$ is infinite, so we may assume otherwise. Consequently, the function $(x, y) \mapsto d_{\mathbb{T}}(x, y)^{-t}$ belongs to $L^{1}(\mu \times \mu)$.

We claim that there is a constant $c$, depending on $t$, such that for all $\varepsilon>0$,

$$
\psi_{\varepsilon} * d_{\mathbb{T}}(\cdot, 0)^{-t}(z) \leq c d_{\mathbb{T}}(z, 0)^{-t} \text { for all } z \in \mathbb{T}^{d} .
$$

One way to show this is to decompose $d_{\mathbb{T}}(\cdot, 0)^{-t}$ as $d_{1}+d_{2}$ where $d_{2}$ equals $d_{\mathbb{T}}(\cdot, 0)^{-t}$ restricted to the complement of a small neighbourhood of 0 . As $d_{2}$ is bounded, $\psi_{\varepsilon} * d_{2}$ is bounded. Clearly $\psi_{\varepsilon} * d_{1}(z)=0$ if $z$ is not sufficiently close to 0 , and one can check that it is bounded by $c d_{\mathbb{T}}(z, 0)^{-t}$ (for a suitable choice of $c$ ) otherwise.

It follows that the functions $(x, y) \mapsto \psi_{\varepsilon} * F_{t}(x-y)$ are all dominated by an integrable function and therefore Lebesgue's theorem gives

$$
\begin{aligned}
\liminf \iint \psi_{\varepsilon} * F_{t}(x-y) d \mu(x) d \mu(y) & =\iint F_{t}(x-y) d \mu(x) d \mu(y) \\
& \leq\|\phi\|_{\infty} I_{t}(\mu)+\left\|g_{t}\right\|_{\infty}|\widehat{\mu}(0)|^{2} .
\end{aligned}
$$

An application of Parseval's theorem as above, together with the observation that $I_{t}(\mu)$ dominates a multiple of $|\widehat{\mu}(0)|^{2}$, completes the proof of the left-hand inequality when $I_{t}(\mu)$ is finite. 
It is known that there are singular measures $\mu$ on the circle with $\{\widehat{\mu}(n)\} \in l^{p}$ for $p>2$. We can give a lower bound on the Hausdorff dimension of such measures.

Corollary 2.3. If $\widehat{\mu} \in l^{p}\left(\mathbb{Z}^{d}\right)$ for some $p>2$, then $\operatorname{dim}_{H} \mu \geq 2 d / p$.

Proof. This follows from Hölder's inequality and the fact that $\sum_{z \in \mathbb{Z}^{d} \backslash\{0\}}|z|^{(t-d) q}$ is finite when $(t-d) q<-d$.

Example 1. Recall that the energy dimension of the Hausdorff measure $\mu$ supported on the standard middle-third Cantor set is $\log 2 / \log 3$. Thus for all $t<$ $\log 2 / \log 3$

$$
|\widehat{\mu}(0)|^{2}+\sum_{n \neq 0}|n|^{t-1}|\widehat{\mu}(n)|^{2} \sim I_{t}(\mu)<\infty .
$$

\section{RiESZ PRODUCTS}

A sequence $\left\{\gamma_{k}\right\} \subseteq \mathbb{Z}^{d}$ is called dissociate if for any positive integer $N$,

$$
\sum_{k=1}^{N} \varepsilon_{k} \gamma_{k}=0 \text { for } \varepsilon_{k}=0, \pm 1, \pm 2 \text { implies } \varepsilon_{k}=0 \text { for all } k .
$$

A lacunary sequence of positive integers $\left\{n_{k}\right\}$ with $n_{k+1} / n_{k} \geq 3$ is an example of a dissociate sequence in $\mathbb{Z}$.

Given a dissociate sequence $\left\{\gamma_{k}\right\}$ and a sequence of complex numbers $\left\{a_{k}\right\}$ satisfying $\sup _{k}\left|a_{k}\right| \leq 1$, we define trigonometric polynomials

$$
P_{k}(x)=\prod_{j=1}^{k}\left(1+\operatorname{Re} a_{j} e^{i \gamma_{j} \cdot x}\right)
$$

for $x \in \mathbb{T}^{d}$. By a Riesz product measure

$$
\mu_{\left\{a_{j}\right\}} \equiv \prod_{j=1}^{\infty}\left(1+\operatorname{Re} a_{j} e^{i \gamma_{j} \cdot x}\right)
$$

we mean the weak ${ }^{*}$ limit of the measures $P_{k}(x) d x$ on $\mathbb{T}^{d}$, which we identify here with $[-\pi, \pi]^{d}$.

3.1. Hausdorff dimension. Estimates of the Hausdorff dimension of Riesz products on $\mathbb{T}$ were first obtained by Peyrière in 11 using probabilistic ideas. He proved that if $n_{k+1} / n_{k} \in \mathbb{Z}$ and $n_{k+1} / n_{k} \geq 3$, then the Hausdorff dimension of the Riesz product measure $\mu_{\left\{a_{j}\right\}}=\prod_{j=1}^{\infty}\left(1+\operatorname{Re} a_{j} e^{i n_{j} x}\right)$ satisfies

$$
\begin{aligned}
& 1-\liminf _{k \rightarrow \infty}\left(\frac{\int \log P_{k} d \mu_{\left\{a_{j}\right\}}}{\log n_{k+1}}\right) \\
& \quad \geq \operatorname{dim}_{H} \mu_{\left\{a_{j}\right\}} \geq 1-\limsup _{k \rightarrow \infty}\left(\frac{\int \log P_{k} d \mu_{\left\{a_{j}\right\}}}{\log n_{k}}\right)
\end{aligned}
$$

([11, Thm. 2.8]). From this formula and (the proof of) [11 Lemma 2.3], one can obtain an upper bound on the Hausdorff dimension of $\mu_{\left\{a_{j}\right\}}$ in terms of the size 
of the coefficients $\left\{a_{j}\right\}$ and the integers $\left\{n_{j}\right\}$. When the coefficients are small in modulus, the Hausdorff dimension of $\mu_{\left\{a_{j}\right\}}$ is approximately bounded above by

$$
1-\liminf _{k \rightarrow \infty}\left(\frac{1}{4} \sum_{j=1}^{k}\left|a_{j}\right|^{2} / \log n_{k}\right) .
$$

In [2, Brown et al expanded the class of Riesz product measures to which Peyrière's integral formula (3.1) applied, replacing the divisibility condition by a less restrictive technical condition, and showed that the Hausdorff dimension of these Riesz products was bounded below by

$$
1-\limsup _{k \rightarrow \infty}\left(\sum_{j=1}^{k}\left|a_{j}\right| / \log n_{k}\right) .
$$

In the next theorem we use our formula (2.2) relating energy and the Fourier transform to improve this lower bound; the new lower bound (3.5) should be compared with Peyrière's upper bound (3.2).

Theorem 3.1. Suppose $\left\{n_{j}\right\}$ is a dissociate set of increasing, positive integers and assume there is some $c<1$ such that $\sum_{j=1}^{k-1} n_{j} \leq c n_{k}$ for all $k$. Let $\mu_{\left\{a_{j}\right\}}$ be the Riesz product $\prod_{j=1}^{\infty}\left(1+\operatorname{Re} a_{j} e^{i n_{j} x}\right)$. Then the energy dimension of $\mu_{\left\{a_{j}\right\}}$ equals $1-\alpha_{0}$ where

$$
\alpha_{0}=\max \left(\limsup _{k \rightarrow \infty}\left(\frac{2 \log \left|a_{k}\right|+\sum_{j=1}^{k-1} \log \left(1+\left|a_{j}\right|^{2} / 2\right)}{\log n_{k}}\right), 0\right) .
$$

In particular,

$$
\operatorname{dim}_{H} \mu_{\left\{a_{j}\right\}} \geq 1-\limsup _{k \rightarrow \infty}\left(\frac{1}{2} \sum_{j=1}^{k}\left|a_{j}\right|^{2} / \log n_{k}\right) .
$$

Before proving this, there are several observations we would like to make.

First, note that the technical assumption of our theorem, the condition that $\sum_{j=1}^{k-1} n_{j} \leq c n_{k}$ for some $c<1$, is automatically satisfied when $n_{k+1} / n_{k} \geq 3$, a necessary condition in both [11] and [2].

In the special case $a_{j}=a \geq 0$ for all $j, n_{j}=q^{j}$ for some integer $q \geq 3$ and $\mu_{a}=\prod_{j=1}^{\infty}\left(1+a \operatorname{Re} e^{i q^{j} x}\right)$ there is an exact (theoretical) formula for the Hausdorff dimension given in [5]:

$$
\operatorname{dim}_{H} \mu_{a}=1-\frac{\int \log \left(1+\operatorname{Re} a e^{i q x}\right) d \mu_{a}}{\log q} .
$$

There are approximations for this in terms of $a$ and $q$ (see [6]); however, our theorem appears to easily give improved estimates.

Corollary 3.2. If $\mu_{a}=\prod_{j=1}^{\infty}\left(1+\operatorname{Re} a e^{i q^{j} x}\right)$, then $\operatorname{dim}_{H} \mu_{a} \geq 1-\left|a^{2}\right| /(2 \log q)$. More generally, if $\log n_{k} / k \rightarrow \log q<\infty$ and $\mu_{\left\{a_{j}\right\}}=\prod_{j=1}^{\infty}\left(1+\operatorname{Re} a_{j} e^{i n_{j} x}\right)$, then

$$
\operatorname{dim}_{H} \mu_{\left\{a_{j}\right\}} \geq 1-\frac{\limsup \sup _{k} \frac{1}{k} \sum_{j=1}^{k}\left|a_{j}\right|^{2}}{2 \log q} .
$$


In [11] Peyrière gave examples of Riesz products satisfying $n_{k+1} / n_{k} \rightarrow \infty$ which had Hausdorff dimension one. In fact, another consequence of our theorem is that all such Riesz products have dimension one.

Corollary 3.3. (a) If $k / \log n_{k} \rightarrow 0$, then $\operatorname{dim}_{H} \mu_{\left\{a_{j}\right\}}=1$.

(b) If $n_{k+1} / n_{k} \rightarrow \infty$, then $\operatorname{dim}_{H} \mu_{\left\{a_{j}\right\}}=1$.

Proof. (a) Since $\sum_{j=1}^{k}\left|a_{j}\right|^{2} \leq k$, the hypothesis implies $\sum_{j=1}^{k}\left|a_{j}\right|^{2} / \log n_{k} \rightarrow 0$.

(b) Given $\varepsilon>0$, choose $m$ such that $\log m>1 / \varepsilon$ and pick $k(m)$ so that for all $k>k(m), n_{k+1} / n_{k}>m$. If $k=k(m)+j$, then $n_{k} \geq m^{j} n_{k(m)}$ and therefore if $j$ (equivalently, $k$ ) is sufficiently large, then

$$
\frac{k}{\log n_{k}} \leq \frac{k(m)+j}{j \log m} \leq 2 \varepsilon
$$

Proof. To calculate the energy dimension of $\mu_{\left\{a_{j}\right\}}$ (we will write $\mu$ in what follows) we need to observe that if

$$
n \in \Gamma_{k} \equiv\left\{ \pm n_{k}+\sum_{j=1}^{k-1} \varepsilon_{j} n_{j}: \varepsilon_{j}=0, \pm 1\right\},
$$

then

$$
|\widehat{\mu}(n)|=\frac{\left|a_{k}\right|}{2} \prod_{j: \varepsilon_{j} \neq 0} \frac{\left|a_{j}\right|}{2}
$$

(where the empty product is one). Furthermore, if $n \in \Gamma_{k}$, then $|n| \sim n_{k}$. Thus,

$$
\sum_{n \in \Gamma_{k}}|n|^{-\alpha}|\widehat{\mu}(n)|^{2} \sim n_{k}^{-\alpha} \frac{\left|a_{k}\right|^{2}}{2} \prod_{j=1}^{k-1}\left(1+\frac{\left|a_{j}\right|^{2}}{2}\right)
$$

and therefore

$$
I_{1-\alpha}(\mu) \sim 1+\sum_{k=1}^{\infty} n_{k}^{-\alpha} \frac{\left|a_{k}\right|^{2}}{2} \prod_{j=1}^{k-1}\left(1+\frac{\left|a_{j}\right|^{2}}{2}\right) .
$$

Clearly, $I_{1-\alpha}(\mu)=\infty$ if infinitely many of the summands are at least one, and this occurs if

$$
-\alpha \log n_{k}+\log \frac{\left|a_{k}\right|^{2}}{2}+\sum_{j=1}^{k-1} \log \left(1+\frac{\left|a_{j}\right|^{2}}{2}\right) \geq 0
$$

for infinitely many $k$. It is a routine verification to see that if $\alpha<\alpha_{0}$, then this is indeed the case.

Conversely, $I_{1-\alpha}(\mu)<\infty$ if there is some $A<1$ such that for all but finitely many $k$,

$$
n_{k}^{-\alpha} \frac{\left|a_{k}\right|^{2}}{2} \prod_{j=1}^{k-1}\left(1+\frac{\left|a_{j}\right|^{2}}{2}\right) \leq A^{k}
$$

or, equivalently,

$$
\alpha \geq \frac{\log \left|a_{k}\right|^{2} / 2+\sum_{j=1}^{k-1} \log \left(1+\left|a_{j}\right|^{2} / 2\right)+k|\log A|}{\log n_{k}} .
$$


It is known ([12]) that if $\left\{n_{j}\right\}$ is a dissociate sequence of positive integers, then

$$
\left|\left\{n_{j}\right\} \bigcap\left[1,2^{k}\right]\right| \leq O(k)
$$

hence, there must be some $C>0$ such that $n_{k} \geq 2^{C k}$ for all $k$. Thus it suffices to show that for all but finitely many $k$,

$$
\alpha \geq \frac{\log \left|a_{k}\right|^{2} / 2+\sum_{j=1}^{k-1} \log \left(1+\left|a_{j}\right|^{2} / 2\right)}{\log n_{k}}+\frac{|\log A|}{C \log 2}
$$

for some $A<1$. We can certainly achieve this if $\alpha>\alpha_{0}$ since we can choose $A$ so close to 1 that $|\log A| / C \log 2$ is as small as necessary.

Since $\log (1+x) \leq x$ for $x>0$, inequality (3.5) follows directly.

Remark 3.1. Similar results can obviously be proved for Riesz products

$$
\prod_{j=1}^{\infty}\left(1+\operatorname{Re} a_{j} e^{i \gamma_{j} \cdot x}\right)
$$

in $\mathbb{T}^{d}$ where $\left\{\gamma_{j}\right\} \subseteq \mathbb{Z}^{d}$ is dissociate and satisfies $\sum_{j=1}^{k-1}\left|\gamma_{j}\right| \leq c\left|\gamma_{k}\right|$ for some $c<1$.

3.2. Random Riesz products. One can similarly define a random Riesz product as the weak* limit of the sequence of measures

$$
\prod_{j=1}^{k}\left(1+\operatorname{Re} a_{j} e^{i \gamma_{j} \cdot\left(x+\omega_{j}\right)}\right) d x
$$

where $\omega=\left\{\omega_{j}\right\}$ is a sequence of independent and identically distributed, random variables on $\mathbb{T}^{d}$.

The results of Peyrière and Brown et al were extended by Fan in 5 to random Riesz products. He showed, for example, that if $n_{k+1} / n_{k} \geq 3, \lim \left(k / \log n_{k}\right)=\xi$ exists and $a_{k}=a \in \mathbb{R}$, then the Hausdorff dimension of the random Riesz product $\mu_{a, \omega}=\prod_{j=1}^{\infty}\left(1+a \operatorname{Re} e^{i \gamma_{j} \cdot\left(x+\omega_{j}\right)}\right)$ equals

$$
1-\xi\left(1-\sqrt{1-a^{2}}+\log \left(\left(1+\sqrt{1-a^{2}}\right) / 2\right)\right) .
$$

In contrast, we can show that the energy dimension is generally smaller.

Proposition 3.4. If $n_{k+1} / n_{k} \geq 3, \lim _{k \rightarrow \infty}\left(k / \log n_{k}\right)=\xi \neq 0$ and $a_{k}=a \in \mathbb{R}$, then almost surely the Hausdorff dimension of $\mu_{a, \omega}$ exceeds the energy dimension.

Proof. As $\left|\widehat{\mu_{a, \omega}}(n)\right|=\left|\widehat{\mu_{a}}(n)\right|$, formula (3.6) gives that

$$
I_{1-\alpha}\left(\mu_{a, \omega}\right) \sim 1+\frac{a^{2}}{2} \sum_{k=1}^{\infty} n_{k}^{-\alpha}\left(1+\frac{a^{2}}{2}\right)^{k-1} .
$$

Thus the $(1-\alpha)$-energy is infinite if, for infinitely many $k$,

$$
\alpha \leq \frac{(k-1) \log \left(1+a^{2} / 2\right)}{\log n_{k}} \rightarrow \xi \log \left(1+a^{2} / 2\right) .
$$

Since a simple calculus argument shows that

$$
\log \left(1+a^{2} / 2\right)>1-\sqrt{1-a^{2}}+\log \left(\left(1+\sqrt{1-a^{2}}\right) / 2\right) \text { if } a \neq 0
$$

the result is immediate. 
3.3. Riesz products on $\mathbb{R}^{d}$. Any measure $\mu$ on $\mathbb{T}^{d}$ (which is now convenient to identify with $[-1 / 2,1 / 2]^{d}$ ) can be extended periodically to $\mu_{e}$ on $\mathbb{R}^{d}$, the extension $\mu_{e}$ being a measure which is infinite except in the trivial case when $\mu=0$. Let $\phi$ be any strictly positive, $C^{\infty}\left(\mathbb{R}^{d}\right)$ function, whose Fourier transform is a positive function supported in $B(0,1 / 2)$ and satisfying $\widehat{\phi}(0)>0$. Assume also that $\phi$ decays sufficiently rapidly to ensure that $\phi \mu_{e}$ is a finite measure.

Following Peyrière, we will say that $\nu$ is a Riesz product measure on $\mathbb{R}^{d}$ if $\nu=\phi \mu_{e}$ where $\mu$ is a Riesz product on $\mathbb{T}^{d}$ and $\phi$ is as above.

Proposition 3.5. Suppose $\mu$ is a measure on $\mathbb{T}^{d}$ and $\phi \mu_{e}$ is a finite measure on $\mathbb{R}^{d}$ where $\mu_{e}$ is the periodic extension of $\mu$ to $\mathbb{R}^{d}$ and $\phi$ is as described above. Then $I_{t}(\mu)$ is comparable to $I_{t}\left(\phi \mu_{e}\right)$.

Proof. Formally, the periodic extension $\mu_{e}$ of $\mu$ is the convolution of the compactly supported measure $\mu$ defined on $[-1 / 2,1 / 2]^{d} \subseteq \mathbb{R}^{d}$, with the distribution $P=$ $\sum_{a \in \mathbb{Z}^{d}} \delta_{a}$ where $\delta_{a}$ is the point mass measure at $a \in \mathbb{R}^{d}$. Hörmander's theorem ([7] 7.2.1]) gives $\widehat{P}=P$; thus, $\widehat{\mu_{e}}$ is the distribution $\widehat{\mu * P}=\sum_{a \in \mathbb{Z}^{d}} \widehat{\mu}(\cdot) \delta_{a}$.

Let $\nu=\phi \mu_{e}$ where $\phi$ is as above. Since $\nu$ is a finite measure on $\mathbb{R}^{d}$ the classical formula can be applied:

$$
I_{t}(\nu)=c \int|x|^{t-d}|\widehat{\nu}(x)|^{2} d x .
$$

Now $\widehat{\nu}(x)=\widehat{\mu_{e}} * \widehat{\phi}(x)=\sum_{a \in \mathbb{Z}^{d}} \widehat{\mu}(a) \widehat{\phi}(x-a)$, and as supp $\widehat{\phi} \subseteq B(0,1 / 2)$, there can be at most one nonzero term in the sum for any given $x$. Thus we can decompose the integral above as

$$
\begin{aligned}
I_{t}(\nu) & =c \sum_{a \in \mathbb{Z}^{d}}|\widehat{\mu}(a)|^{2} \int_{B(0,1 / 2)}|\widehat{\phi}(x)|^{2}|x+a|^{t-d} d x \\
& \sim|\widehat{\mu}(0)|^{2}+\sum_{a \in \mathbb{Z}^{d} \backslash\{0\}}|\widehat{\mu}(a)|^{2}|a|^{t-d}
\end{aligned}
$$

since $\widehat{\phi}$ is bounded away from zero in a neighbourhood of the origin. But this is comparable to $I_{t}(\mu)$ according to our formula (2.2).

Corollary 3.6. If $\nu=\phi \mu_{e}$ is a Riesz product measure on $\mathbb{R}^{d}$ corresponding to the Riesz product $\mu$ on $\mathbb{T}^{d}$, then the energy dimensions of $\nu$ and $\mu$ are equal.

Remark 3.2. The Hausdorff dimensions of $\nu$ and $\mu$ are also equal since $\phi \mu_{e}$ and $\mu_{e}$ have the same null sets.

\section{REFERENCES}

1. J. Bourgain, Hausdorff dimension and distance sets, Israel J. Math. 87 (1994), 193-201. MR 95h:28008

2. G. Brown, W. Moran, and C. Pearce, Riesz products, Hausdorff dimension and normal numbers, Math. Proc. Camb. Phil. Soc. 101 (1987), 529-540. MR 88f:42024

3. K. Falconer, Fractal geometry. Mathematical foundations and applications, Wiley and Sons, Chichester, 1990. MR 92j:28008

4. K. Falconer, Techniques in fractal geometry, Wiley and Sons, Chichester, 1997. MR 99f:28013

5. Ai Hua Fan, Quelques propriétés des produits de Riesz, Bull. Sci. Math. 117 (1993), 421-439. MR 95f:28002

6. Ai Hua Fan, Une formule approximative de dimension pour certains produits de Riesz, Monatsh. Math. 118 (1994), 83-89. MR 96f:42006 
7. L. Hörmander, The analysis of linear partial differential operators I, Springer-Verlag, Berlin, 1983. MR 85g:35002a

8. P. Mattila, Geometry of sets and measures in Euclidean spaces, Cambridge Studies in Advanced Mathematics 44, Cambridge Univ. Press, Cambridge, 1995. MR 96h:28006

9. P. Mattila, Spherical averages of Fourier transforms of measures with finite energy; dimension of intersections and distance sets, Mathematica 34 (1987), 207-228. MR 90a:42009

10. Y. Peres and W. Schlag, Smoothness of projections, Bernoulli convolutions and the dimensions of exceptions, Duke Math. J. 102 (2000), 193-251. MR 2001d:42013

11. J. Peyrière, Etude de quelques propriétés des produits de Riesz, Ann. Inst. Fourier, Grenoble 25 (1975), 127-169. MR 53:8771

12. W. Rudin, Trigonometric series with gaps, J. Math. Mech. 9 (1960), 203-227. MR 22:6972

13. P. Sjölin and F. Soria, Some remarks on restrictions of the Fourier transform for general measures, Publicacions Math. 43 (1999), 655-664. MR 2001i:42017

14. A. Zygmund, Trigonometric series I, Cambridge Univ. Press, Cambridge, 1959. MR 21:6498

Department of Pure Mathematics, University of Waterloo, Waterloo, Ontario, CANADA N2L 3G1

E-mail address: kehare@uwaterloo.ca

Department of Mathematics, Chalmers th and Goteborg University, Eklandagatan 86, SE-41296, SWEDEN

E-mail address: maria@math.chalmers.se 